DOI: http://dx.doi.org/10.12957/demetra.2014.11979

\title{
Avaliação das rotulagens e informações nutricionais dos pães integrais: fibras, sódio e adequação com a legislação vigente
}

\section{Evaluation of labeling and nutritional information of wholemeal breads: fiber, sodium and adequacy with current legislation}

\author{
Vadete Carla Pissaia da Silva' \\ Carin Weirich Gallon' \\ Heloísa Theodoro' \\ ' Curso de Nutrição, Universidade de Caxias do \\ Sul. Caxias do Sul-RS, Brasil. \\ Correspondência / Correspondence \\ Vadete Carla Pissaia da Silva \\ E-mail: pissaiavs@yahoo.com.br
}

\section{Resumo}

A garantia de informações úteis e confiáveis em rotulagem de alimentos é um direito assegurado pelo Código de Proteção e Defesa do Consumidor que colabora na escolha de melhores produtos. Este estudo teve como objetivo avaliar as rotulagens e informações nutricionais dos pães integrais quanto a sua composição de fibras e sódio, adequação às legislações vigentes e com o Substitutivo da $\mathrm{Lei}^{\circ} 5.081 / 2013$, que está tramitando no Congresso Nacional. Trata-se de estudo observacional e descritivo realizado na cidade de Caxias do Sul-RS, cuja coleta de dados ocorreu de abril a maio de 2014. Analisaram-se 30 rótulos de pães integrais e aplicou-se um formulário construído com base nos objetivos propostos: quantidade de fibras e sódio em $50 \mathrm{~g}$, adequação à RDC 90, RDC 54 e ao Substitutivo, o primeiro ingrediente ser farinha integral, preço e existência do selo de qualidade, o qual certifica produtos integrais. Constatou-se que, de todos os rótulos avaliados, metade está inadequada quanto à RDC $\mathrm{n}^{\mathrm{o}} 54 ; 73,3 \%$ possuem irregularidade quanto à RDC $\mathrm{n}^{\mathrm{o}}$ 90; 73,3\% não estão adequados ao Substitutivo; $60 \%$ das marcas não possuíam como primeiro ingrediente a farinha integral; e $86,7 \%$ das marcas verificadas não possuem o selo de certificação. A média de sódio encontrada nos rótulos foi de 185 miligramas, o que representa mais do dobro do que é considerado "baixo teor de sódio" referido na RDC n⿳0 54. Conclui-se que os rótulos avaliados neste estudo, em sua maioria, apresentaram diversas irregularidades em relação aos objetivos propostos.

Palavras-chave: Rotulagem dos alimentos. Fibras. Sódio. Informação Nutricional. Legislação sobre pães integrais. Legislação sobre Alimentos. Pão. 


\section{Abstract}

Ensuring useful and reliable information on food labels is a right secured by the Code of Consumer Protection that helps choose the best products. This study aimed to evaluate the labeling and nutritional information of wholemeal breads concerning composition of fiber and sodium, according to the adequacy of existing laws and the Substitute of Law 5.081/2013, pending in Congress. This is an observational and descriptive study conducted in Caxias do Sul-RS, with data collected from April to May 2014. We analyzed 30 labels of breads and applied a form based on the proposed objectives: amount of fiber and $50 \mathrm{~g}$ sodium, fitness for RDC 90, RDC 54, and Substitute, the first ingredient is whole wheat flour, price and availability of the quality seal that certifies wholegrain products. It was found that, of all labels evaluated, half is inappropriate to RDC No. $54 ; 73.3 \%$ are irregular with regard to RDC No. 90; and $73.3 \%$ are not suitable to the Substitute in process; $60 \%$ of the marks did not have whole wheat flour as first ingredient; and $86.7 \%$ of those seen brands do not have the seal of accreditation. The average sodium found on the labels was $185 \mathrm{mg}$, which represents more than double the "low" attribute referred to the RDC 54. We concluded that most labels in this study showed various irregularities in relation to the proposed objectives.

Key words: Food labeling. Fibers. Sodium. Nutritional Facts. Legislation breads. Legislation Food. Bread.

\section{Introdução}

Um dos alimentos mais recomendados pelos profissionais de saúde é o pão integral. Os grãos integrais são alimentos muito estudados devido às quantidades de fibras que os compõem, porque o consumo adequado de fibras, na dieta, reduz o risco de hipertensão arterial, ${ }^{1}$ diabetes mellito, ${ }^{2,3}$ dislipidemia, ${ }^{4}$ doenças cardiovasculares,${ }^{5}$ doenças gastrointestinais,,${ }^{6,7}$ câncer ${ }^{8-12}$ e obesidade. ${ }^{4,13}$

A farinha de trigo integral para uso doméstico é obtida "a partir de cereal limpo e com teor máximo de cinzas de 2,0\% na base seca". Para uso industrial, utiliza-se a farinha obtida a partir do cereal limpo e com teor máximo de cinzas de 2,5\% na base seca, devendo obedecer aos requisitos específicos para cada segmento de aplicação. ${ }^{14}$ 
A American Association of Cereal Chemists International (AACCI) e a Food and Drug Administration (FDA) definem os grãos integrais "como a parte intacta do grão, cujo principais componentes, o endosperma, gérmen e farelo, estão presentes nas mesmas proporções relativas com grão intacto". ${ }^{15,16}$ As farinhas integrais consistem de grãos moídos em sua totalidade e são constituídos pelo farelo, pelo gérmen e pelo endosperma. ${ }^{17}$

O pão integral, segundo a Resolução-RDC n⿳0 90, de 18 de outubro de 2000, é definido como "produto preparado, obrigatoriamente, com farinha de trigo e farinha de trigo integral e/ou fibra de trigo e ou farelo de trigo"18 No entanto, devido à falta do estabelecimento de quantidades mínimas de farinha integral, qualquer quantidade, mesmo que ínfima, a rotulagem alegará como sendo farinha integral em sua composição.

A prevalência de consumo de pão integral, segundo sexo, está representada em masculino $(1,3 \%)$ e feminino (2,4\%). A Região Sul apresenta o maior percentual de consumo alimentar, com uma taxa de 4,3\%. O consumo alimentar per capita por classes de rendimento está entre salários maiores de R\$1.089,00, o que representa 2,5 gramas $(\mathrm{g}) / \mathrm{dia} .{ }^{19}$

O Instituto Brasileiro de Geografia e Estatística (IBGE), em parceria com o Ministério da Saúde, analisou o perfil nutricional do país e verificou que $68 \%$ da população consomem pouca fibra, ficando abaixo do recomendado, e que mais de $70 \%$ da população consomem valores acima da ingestão tolerável para o sódio, ${ }^{19}$ o que é confirmado pela média de consumo da população brasileira, que é de 9,6g/dia/per capita..$^{20}$ A Organização Mundial da Saúde (OMS) recomenda quantidades inferiores a 2.000 miligramas (mg), como limite de consumo de sódio por dia, sendo que os hipertensos devem consumir quantidades abaixo desta recomendação. ${ }^{21} \mathrm{O}$ sódio em excesso é considerado causa da hipertensão arterial, câncer de estômago e de acidente vascular cerebral. ${ }^{20} \mathrm{O}$ perfil nutricional do brasileiro acima exposto pode ser explicado pelo alto consumo de alimentos industrializados.

A globalização, a urbanização e a inserção da mulher no mercado de trabalho são fatores que colaboraram para a mudança no padrão alimentar do brasileiro. Com a globalização, houve oferta de produtos antes não consumidos; e a urbanização (no Brasil, entre os anos de 2000-2010, houve aumento de 23 milhões de pessoas) fizeram com que os centros urbanos fossem locais de interesse das redes de supermercados, favorecendo o acesso a alimentos prontos, ricos em sódio, gordura e açúcares. ${ }^{22}$ Os alimentos preparados, nos últimos 30 anos, tiveram aumento de consumo em $317,64 \% .^{23}$

Nos dias de hoje, há vasta oferta de alimentos industrializados "ultraprocessados", que Monteiro et al. ${ }^{24}$ definem como "todo alimento derivado de matéria-prima alimentar ou de alimento in natura, adicionado ou não de outros alimentos processados, com elevado grau de processamento e maior 
durabilidade, acessibilidade e palatabilidade”. Pode ter adição de sal e açúcar, aditivos, vitaminas e minerais, podendo ser submetido a técnicas como fritar, assar, defumar, refogar, cozinhar ou secar. São geralmente alimentos com altas quantidades de energia, sódio, gorduras trans, bem como com poucas fibras, que, segundo Popkin, têm forte relação com o aumento de doenças crônicas não transmissíveis. ${ }^{25}$ São exemplos de alimentos industrializados ultraprocessados: pães de forma, produtos congelados e prontos, como pizzas, massas, hambúrgueres, nuggets, salsichas e outros embutidos, produtos panificados que possuem gordura vegetal hidrogenada, emulsificantes e outros aditivos, biscoitos, sorvetes, bolos e misturas para bolo, salgadinhos "de pacote", balas e guloseimas em geral, macarrão e temperos "instantâneos", refrescos refrigerantes entre outros. ${ }^{26}$

O IBGE verificou que misturas industrializadas e refeições prontas cresceram em $37 \% .{ }^{19} \mathrm{~A}$ procura por alimentos práticos e rápidos pode ser parcialmente explicada por outro fator que colaborou para o aumento do consumo de alimentos industrializados: a inserção das mulheres no mercado de trabalho. Isto causou escassez de tempo na preparação das refeições, aumentando o consumo de lanches, alimentos prontos e fast foods. ${ }^{22} \mathrm{O}$ marketing exagerado de alimentos calóricos e pobres em nutrientes e com preços acessíveis, ${ }^{27}$ assim como a praticidade de não necessitar de utensílios como talheres e pratos, favoreceu a opção por alimentos industrializados. ${ }^{26}$

Considerando estes fatores, é necessário utilizar e conhecer as informações que constam nos rótulos dos alimentos, para que os consumidores possam escolher os melhores produtos e os profissionais da saúde possam indicar os alimentos que possuam maiores benefícios à população.

O objetivo deste estudo foi avaliar as rotulagens e as informações nutricionais dos pães integrais quanto a sua composição de fibras e sódio, bem como quanto à adequação de acordo com as legislações vigentes.

\section{Metodologia}

Trata-se de estudo observacional e descritivo, de avaliação das rotulagens de diferentes marcas de pães integrais. Foram escolhidos 30 pães comercializados em Caxias do Sul, estado do Rio Grande do Sul. As marcas dos pães integrais analisadas não foram identificadas, a fim de preservar sua identidade. Para comparação entre as variedades de produtos, os diferentes pães foram denominados por números. Os critérios de inclusão foram que as rotulagens possuíssem a expressão "pão integral”; constassem na Informação Nutricional, as quantidades de fibras e sódio; e que a porção fosse de $50 \mathrm{~g}$. Esta quantidade de porção dos pães foi baseada na RDC 359, ${ }^{28}$ de 23 
de dezembro de 2003, que determina a porção de $50 \mathrm{~g}$ para os pães embalados, fatiados ou não, com ou sem recheio.

Foram excluídos os pães que não possuíam, na rotulagem, a expressão "pão integral” e que contivessem porção diferente de $50 \mathrm{~g}$. Foram coletadas informações sobre a quantidade de fibras, assim como de sódio, a cada $50 \mathrm{~g}$ de pão contido na rotulagem. Na análise dos rótulos dos alimentos dos pães integrais, utilizou-se um formulário, construído com base nos objetivos propostos: quantidades de sódio e fibras em $50 \mathrm{~g}$, adequação dos termos utilizados na rotulagem, segundo RDC n⿳ 54, de 12 de novembro de 2012, que define fonte de fibras ou alto conteúdo de fibras. Avaliou-se, também, se as empresas estão se adequando ao Substitutivo ao Projeto de Lei n⿳ 5.081/2013, o qual dispõe sobre a comercialização dos pães integrais, assim como o primeiro ingrediente mencionado na informação nutricional, existência do selo de certificação de produtos integrais e preço.

O processo de seleção das marcas foi realizado no período de abril a maio de 2014. Foram registrados os dados em banco de dados para posterior análise descritiva e comparações entre as amostras e variáveis. Neste estudo, utilizou-se a estatística descritiva, no qual os dados foram representados por meio de números absolutos e frequência, e também por média e desvio padrão. Os dados foram analisados no programa estatístico Statistical Package for Social Sciences $(\mathrm{SPSS})^{\circledR}$ versão 18.0. Utilizaram-se os testes de qui quadrado e $t$ de Student. Os resultados foram considerados estatisticamente significativos quando apresentaram o valor de $p$ menor ou igual a 0,05 .

\section{Resultados}

Ao total, foram analisados 30 rótulos de pães integrais. A partir da análise dos rótulos alimentares, constataram-se algumas inadequações quanto às legislações vigentes, assim como ao Substitutivo que está tramitando no Senado. Observou-se que metade dos produtos não estava adequada à RDC n⿳0 54/2012, relativa à adequação dos termos "fonte" e "alto conteúdo" de fibras. Em torno de 73,3\% dos rótulos não apresentam adequação quanto ao Substitutivo. E ainda, em 73,3\% deles não estava declarado o percentual de farinha integral, conforme o item 9.4 da RDC 90, de outubro de 2000. As demais variáveis relacionadas à legislação encontram-se dispostas na tabela 1 . 
Tabela 1. Percentual de Adequação à Legislação Vigente em relação aos rótulos avaliados de pães integrais em supermercados do município de Caxias do Sul-RS, 2014.

\begin{tabular}{cc}
\hline Variável & $\mathrm{N}(\%)$ \\
\hline $\begin{array}{c}\text { Adequação termos fonte e alto conteúdo } \\
\text { de fibras RDC no 54/201224 }(\mathrm{n}=30)\end{array}$ & $15(50,0)$ \\
Não & $15(50,0)$ \\
Adequação Fonte $(\mathrm{n}=14)$ & $2(14,3)$ \\
Não & $12(85,7)$ \\
Sim & \\
Adequação \% farinha integral > 51\% ou & $22(73,3)$ \\
$<51 \%$ - Lei no $5.08126(\mathrm{n}=30)$ & $8(26,7)$ \\
Não & \\
Sim & $22(73,3)$ \\
undilizada & $8(26,7)$ \\
Não declarado & \\
Declarado &
\end{tabular}

Legenda : n= número; RDC= Resolução da Diretoria Colegiada; $>=$ maior; $<=$ menor.

Observou-se que, dos rótulos que apresentam o selo de certificação, apenas 33,3\% apresentam, como primeiro ingrediente, a farinha integral. Quando analisada a relação entre a existência do selo de qualidade e a adequação ao percentual de farinha integral, encontrou-se significância estatística - ou seja, os rótulos que possuem o selo são os que mais estão adequados às legislações (tabela 2). 
Tabela 2. Relação entre a existência entre o selo de certificação e adequações dos rótulos avaliados de pães integrais em supermercados do município de Caxias do Sul-RS, 2014 ..

\begin{tabular}{|c|c|c|}
\hline Variável & Selo adequado (\%) & $\mathrm{P}$ valor* \\
\hline \multicolumn{3}{|c|}{ Adequação termos fonte e alto conteúdo } \\
\hline \multicolumn{3}{|l|}{ de fibras } \\
\hline \multicolumn{3}{|l|}{ RDC n 54/201224 } \\
\hline Não & 6,7 & \multirow[t]{2}{*}{0,59} \\
\hline Sim & 20,0 & \\
\hline \multicolumn{3}{|c|}{ Adequação Fonte $(\mathrm{n}=14)$} \\
\hline Não & 0 & \multirow[t]{2}{*}{1,0} \\
\hline $\operatorname{Sim}$ & 25,0 & \\
\hline \multirow{2}{*}{\multicolumn{3}{|c|}{$\begin{array}{c}\text { Adequação } \% \text { farinha integral }>51 \% \text { ou } \\
<51 \% \text { Lei no } 5.08126\end{array}$}} \\
\hline & & \\
\hline$(\mathrm{n}=30)$ & & \multirow[t]{3}{*}{$0,003 *$} \\
\hline Não & 0 & \\
\hline Sim & 50,0 & \\
\hline \multicolumn{3}{|c|}{$\begin{array}{l}\text { Farinha integral-10 na lista de } \\
\text { ingredientes }(\mathrm{n}=30)\end{array}$} \\
\hline Não & 0 & \multirow{3}{*}{$0,02 *$} \\
\hline $\operatorname{Sim}$ & 33,3 & \\
\hline \multicolumn{2}{|c|}{$\begin{array}{c}\text { RDC nº 9020-Declaração \% farinha } \\
\text { utilizada }\end{array}$} & \\
\hline Não declarado & 0 & \\
\hline Declarado & 50,0 & $0,003 *$ \\
\hline
\end{tabular}

Legenda: RDC= Resolução da Diretoria Colegiada; \%= percentual; $\mathrm{n}=$ número; $>=$ maior; $<=$ menor; $1^{\mathrm{o}}=$ primeiro; $\mathrm{p}=$ valor $\mathrm{p}$.

Na relação de preço com outras variáveis analisadas, observou-se que a maioria dos produtos adequados às legislações possui preço mais alto, com significância estatística para as adequações ao Substitutivo e à RDC no 90 (tabela 3). 
Tabela 3. Relação entre preço e adequação dos rótulos e selo de certificação de pães integrais avaliados em supermercados do município de Caxias do Sul-RS, 2014.

\begin{tabular}{|c|c|c|}
\hline Variável & $\begin{array}{l}\text { Preço médio\# } \\
\text { R } \$(D P)\end{array}$ & $\mathrm{P}$ valor* \\
\hline \multicolumn{3}{|c|}{$\begin{array}{l}\text { Adequação termos fonte e alto conteúdo de } \\
\text { fibras RDC n⿳0 54/201224 }\end{array}$} \\
\hline Não & $5,88(1,97)$ & $0,006^{*}$ \\
\hline Sim & $7,99(1,94)$ & \\
\hline \multicolumn{3}{|c|}{ Adequação Fonte $(n=14)$} \\
\hline Não & $7,63(0,29)$ & 0,77 \\
\hline Sim & $8,11(2,17)$ & \\
\hline \multicolumn{3}{|c|}{$\begin{array}{c}\text { Adequação } \% \text { farinha integral }>51 \% \text { ou }< \\
\text { 51\% Lei no } 5.08126\end{array}$} \\
\hline Não & $6,43(2,18)$ & $0,04^{*}$ \\
\hline Sim & $8,32(1,67)$ & \\
\hline \multicolumn{3}{|c|}{ Farinha integral-1 ${ }^{\circ}$ na lista de ingredientes } \\
\hline Não & $6,69(2,28)$ & 0,48 \\
\hline Sim & $7,29(2,11)$ & \\
\hline \multicolumn{3}{|c|}{$\begin{array}{c}\text { RDC nº } 9020 \text { Declaração \% farinha } \\
\text { utilizada }\end{array}$} \\
\hline Não declarado & $6,42(2,18)$ & $0,04 *$ \\
\hline Declarado & $8,32(1,67)$ & \\
\hline \multicolumn{3}{|l|}{ Selo de certificação } \\
\hline Não & $6,78(2,29)$ & 0,35 \\
\hline Sim & $7,91(1,35)$ & \\
\hline
\end{tabular}

Legenda: $\mathrm{RDC}=$ Resolução da Diretoria Colegiada; $\mathrm{n}=$ número; \% = percentual; $>$ = maior; $<=$ menor; \# Preço médio em $500 \mathrm{~g}$ de pão; DP = Desvio Padrão; $\mathrm{R} \$=$ valor em reais; $\mathrm{p}=$ valor $\mathrm{p}$. 
Avaliando-se a relação entre a média de fibras e a existência de farinha integral como primeiro ingrediente, encontrou-se teor médio de fibras de $2,7 \mathrm{~g}(\mathrm{DP}=0,8)$ para os que não possuem a farinha como primeiro ingrediente e uma média de $3,3 \mathrm{~g}(\mathrm{DP}=0,6)$ para os que possuem a farinha integral como primeiro ingrediente, sendo estatisticamente significativo $(p=0,05)$. Ou seja, os rótulos que possuíam a farinha integral como primeiro ingrediente na informação nutricional obtiveram média maior de fibras, quando comparados com os rótulos que não possuíam a farinha integral como primeiro ingrediente.

Na correlação entre fibras e miligramas de sódio, a associação encontrada foi inversa - ou seja, à medida que aumenta a quantidade de fibras do produto, reduz-se a quantidade de sódio existente neste, embora isto não tenha sido estatisticamente significativo $\mathrm{p}=0,55$.

\section{Discussão}

O padrão alimentar contemporâneo está relacionado ao facilitado acesso a alimentos industrializados ricos em açúcares, sódio e gorduras, e pobres em fibras, que favorece o crescimento de doenças crônicas não transmissíveis, colaborando para o perfil de morbimortalidade e aumento contínuo da prevalência de excesso de peso no país. ${ }^{29}$ Através da rotulagem, o consumidor deve ter acesso à informação correta sobre os alimentos que escolhe. Considerando esses fatores, é necessário utilizar e conhecer as informações que constam nos rótulos para os consumidores escolherem os melhores produtos e os profissionais da saúde poderem indicar os alimentos que possuam maiores benefícios à população.

Na presente pesquisa, foram encontradas diversas inadequações quanto às legislações vigentes. Segundo a Resolução RDC no 54, de 12 de novembro de 2012, o alimento, para ser classificado com "alto conteúdo de fibras", deve conter, no mínimo, $5 \mathrm{~g}$ por porção. Para ser definido como "fonte de fibras" deve conter, pelo menos, 2,5g por porção (ANVISA).$^{30}$ As empresas abrangidas por esta Resolução tinham o prazo estabelecido para promover as adequações necessárias nos produtos até o dia 1o de janeiro de 2014. Somente metade das marcas verificadas apresentou adequação à nova norma.

A RDC n⿳ 90, de 18 de outubro de 2000, ${ }^{18}$ aprovou o Regulamento Técnico para fixação de identidade e qualidade do pão no que diz respeito ao item 9.4, o qual declara que "quando o produto apresentar em sua composição farinha de trigo integral, fibra de trigo, farelo de trigo e/ou farinha de cereais (exceto trigo), leguminosas, raízes e tubérculos, deve ser declarado, no rótulo, o percentual destes ingredientes". As empresas tinham o prazo de 180 dias, a contar da publicação do regulamento (20 de outubro de 2000), para se adequar à norma. No presente estudo, observou-se um descumprimento em torno de 73,3\%. 
Faltam parâmetros quanto ao percentual de farinha integral que um pão deve conter, já que a Resolução RDC n⿳⺈ 90, de 18 de outubro de 2000, ${ }^{18}$ o define como "produto preparado, obrigatoriamente, com farinha de trigo e farinha de trigo integral e/ou fibra de trigo e/ou farelo de trigo". Não estabelecendo quantidades mínimas de farinha integral, qualquer quantidade, mesmo que ínfima, será alegada pela rotulagem como tendo farinha integral em sua composição.

Diante deste dilema, foi elaborado o Projeto de Lei 5.081-A, de 2013, ${ }^{31}$ o qual dispõe sobre normas de comercialização do pão integral. A Agência Nacional de Vigilância Sanitária (ANVISA), verificando a necessidade de estabelecer critérios sobre a quantidade de farinha integral presente nos alimentos, sugeriu alterações na redação do Projeto de Lei, sendo que este está tramitando no Congresso Nacional em forma de Substitutivo. O Substitutivo dispõe sobre o uso da expressão "integral e afins" na rotulagem de alimentos à base de cereais.

As sugestões da ANVISA, que compõem o Substitutivo, são resumidamente: ${ }^{32}$ abranger todos os produtos à base de cereais não somente o pão integral; substituir a palavra "embalagem" pelo termo técnico adequado "rotulagem", pois a RDC no 259/02, de 20 de setembro de 2002,, ${ }^{33}$ que define embalagem como sendo o "recipiente, o pacote ou a embalagem destinada a garantir a conservação e facilitar o transporte e manuseio dos alimentos". Rotulagem é "toda inscrição, legenda, imagem ou toda a matéria descritiva ou gráfica, escrita, impressa, estampada, gravada, gravada em relevo ou litografada ou colada sobre a embalagem do alimento".

A ANVISA sugeriu, também, a substituição da expressão "grãos integrais" por "farinhas de cereais integrais" e a criação da expressão "integral”, que deve constar na rotulagem, caso o produto contenha mais que $51 \%$ (cinquenta e um por cento) de grãos integrais em sua composição. Produtos que contiverem grãos integrais acima de $15 \%$ (quinze por cento) até $51 \%$ (cinquenta e um por cento) deverão utilizar na rotulagem a expressão "semi-integral ou com adição de farinha integral”. O produto que contiver adição de grão integral inferior a 15\% (quinze por cento) não poderá utilizar nenhuma referência, em sua rotulagem, de que se trata de produto "integral" ou "semi-integral ou com adição de farinha integral", ou qualquer outra expressão que possa induzir o consumidor a constatar que aquele produto seja integral. Este Substitutivo foi aprovado pela Comissão de Desenvolvimento Econômico, Indústria e Comércio e segue para análise das Comissões de Defesa do Consumidor (CDC) e de Constituição e Justiça e Cidadania (CCJC), onde tramita em caráter conclusivo. No momento, está aguardando parecer do relator da CDC.

A título explicativo, neste estudo, o termo "rótulo" foi escolhido devido à definição da RDC 259/02, ${ }^{33}$ de 20 de setembro de 2002, e a decisão do Substitutivo de modificar o termo "embalagem" para "rótulo". Objetivo deste estudo é justamente a leitura da "rotulagem" dos alimentos pesquisados.

Na presente pesquisa, em relação ao Substitutivo da Lei $n^{\circ} 5.081,{ }^{32} 73,3 \%$ não estariam adequadas à lei caso esta estivesse em vigor. Em torno de 26,7\% estariam adequadas, o que pode ser 
explicado devido à obrigatoriedade imposta a dois fabricantes detentores de marcas nacionalmente conhecidas, por decisão do Tribunal de Justiça do Rio de Janeiro (TJRJ), que estabeleceu prazo de 180 dias (notificação em fevereiro de 2014), para informar em seus rótulos de pães integrais qual o real teor de farinha integral na composição dos produtos. Ação Civil Pública, movida pelo promotor Pedro Rubim, da Promotoria de Justiça de Tutela Coletiva e do Consumidor do Ministério Público do Rio de Janeiro (MP-RJ), foi motivada por denúncias de consumidores.

Neste estudo, observou-se que $60 \%(\mathrm{n}=30)$ das amostras não apresentavam a farinha integral como primeiro ingrediente na lista de ingredientes. Tal resultado foi encontrado em um teste realizado pela PROTESTE - Associação de Consumidores, ${ }^{34}$ entidade civil sem fins lucrativos considerada a maior organização de defesa do consumidor da América Latina, que integra também o Sistema Nacional de Defesa do Consumidor (SNDC), coordenado pelo Departamento de Proteção e Defesa do Consumidor (DPDC), órgão vinculado ao Ministério da Justiça. Esta realizou um teste em 2012 com sete marcas de pães integrais, obtendo como resultado que quatro marcas possuíam como primeiro ingrediente a farinha de trigo refinada. O teste foi utilizado como argumentação ao Projeto de Lei que está tramitando em forma de Substitutivo. ${ }^{32}$ Como exposto anteriormente, a RDC $90^{18}$ não especifica a necessidade deste em aparecer como primeiro ingrediente, mas, com base na RDC no 259, ${ }^{33}$ de setembro de 2002, esta estabelece o Regulamento Técnico sobre Rotulagem de Alimentos Embalados, a qual refere: "todos os ingredientes devem constar em ordem decrescente, da respectiva proporção". Sendo assim, pode-se verificar que um pão que se denomina integral, se não possuir farinha integral como primeiro ingrediente, não se estabelecerá como uma boa escolha alimentar.

Em estudo realizado por Pimentel \& Simões ${ }^{35}$ na região norte do Paraná, em relação ao conhecimento sobre os benefícios à saúde associados ao consumo de fibras alimentares, verificouse nível considerado satisfatório de conhecimento dos consumidores naquela região, sendo que o pão integral ficou entre os produtos mais consumidos pelos entrevistados, perfazendo $58 \%$. Essa informação só reforça que os consumidores conhecem os benefícios das fibras, mas ignoram a necessidade de ler o rótulo, as informações como quantidade da mesma e existência ou não da farinha integral como primeiro ingrediente do produto a ser escolhido. ${ }^{35}$

O Whole Grains Council - WGC (Conselho de Grãos Integrais) é um grupo de defesa do consumidor sem fins lucrativos, situado em Boston, Estados Unidos, ${ }^{36}$ juntamente com sua empresa-mãe, Oldways, apoiado por uma coligação única de pequenas e grandes empresas que fabricam e apoiam produtos integrais. O conselho está constantemente trabalhando com agências governamentais, incluindo a FDA, dos Estados Unidos, e United States Department of Agriculture (USDA) para, através das normas do governo, incentivar o consumo de grãos integrais. ${ }^{37} \mathrm{O}$ WGC visa informar consumidores onde comprar e consumir grãos integrais visando à alimentação saudável e à certificação de empresas que produzem comprovadamente esses grãos. 
Em 2005, o WGC implementou um selo para produtos com grãos integrais. Há duas diferentes variedades de selo: o Basic Stamp, que contém pelo menos $8 \mathrm{~g}$ (meia porção de grãos inteiros, mas também pode conter alguns grãos refinados) e o Selo $100 \%$, que significa que todos os seus ingredientes de grãos são integrais. Há uma exigência mínima de $16 \mathrm{~g}$ por porção. Este selo garante aos clientes que os grãos, flocos e farinhas fornecidos são $100 \%$ integrais, garantindo também que durante o processo os grãos não perdem os nutrientes de sua composição. ${ }^{38}$

O selo WGC é um símbolo de embalagem familiar que ajuda consumidores a escolher e comprar produtos integrais. As empresas que desejam utilizar o selo devem ser membros do WGC e devem apresentar informações sobre cada produto qualificado com o conselho. As empresas também assinam um acordo legal de cumprimento de todas as normas e diretrizes do programa Stamp. ${ }^{37}$ Desde março de 2013, mais de 9.200 produtos utilizam o selo WGC em 41 países diferentes e em cinco idiomas. No Brasil, cinco marcas utilizam este selo. ${ }^{39}$

Encontrou-se, neste estudo, que $86,7 \%$ dos rótulos não possuíam o selo que certifica a garantia de grãos integrais em sua composição. Constatou-se, também, que neste estudo a média de fibras encontradas foi de 2,95g $(\mathrm{DP}=0,75)$. A recomendação diária de fibra é de $25 \mathrm{~g} \cdot{ }^{20}$ Segundo a American Diabetes Assocation (ADA), a recomendação diária de fibra é de $25 \mathrm{~g}$ para mulheres e $38 \mathrm{~g}$ para homens, ou $14 \mathrm{~g}$ a cada $1.000 \mathrm{Kcal}$ da dieta, sendo a mesma da população em geral. ${ }^{21}$

Em relação ao sal, sabe-se que sua restrição na dieta é uma medida recomendada não apenas para hipertensos, mas para a população em geral. O brasileiro ingere o dobro da quantidade máxima de sal recomendada. A atual recomendação é de $5 \mathrm{~g}$ diários de sal (o equivalente a 2.000 miligramas de sódio), o que representa, em medida caseira, uma colher de chá de sal. ${ }^{21}$

No Brasil, o maior consumo médio de sódio está distribuído entre o sexo masculino e a faixa etária de 14 a 18 anos, o que representa um consumo de 3.705,6mg seguido pela faixa etária de 19 a 59 anos, do sexo masculino também, permanecendo entre o valor de $3.637,6 \mathrm{mg} .{ }^{19} \mathrm{O}$ Ministério da Saúde recomenda que a quantidade de sódio não ultrapasse $2.300 \mathrm{mg}$ para indivíduos adultos.

A RDC $54^{30}$ estabelece que o sódio, para que possa ser utilizado o atributo "baixo", deve ter como condição um máximo de $80 \mathrm{mg}$ (para porção de 50g); para ter o atributo "muito baixo", tem que possuir no máximo $40 \mathrm{mg}$ de sódio (em porção de $50 \mathrm{~g}$ ); e para o atributo "não contém" deve haver, no máximo, $5 \mathrm{~g}$ de sódio (por porção de $50 \mathrm{~g}$ ). No presente estudo, encontrou-se uma média de sódio de 185,3mg ( $\mathrm{DP}=54,5)$ na porção de 50g. Considerando que, hipoteticamente, uma pessoa se alimenta-se com porções de pães, ao longo do dia terá ingerido 555,9 miligramas de sódio, o que representa 24,34\% da ingestão indicada por dia, de 2.000g, segundo o Ministério da Saúde. A média encontrada no rótulo das amostras foi mais do que o dobro indicado como atributo "baixo", o que demonstra ser mais um fator importante a ser observado no momento da escolha do produto a ser consumido. 
Estudo de Orlando et al. realizado na região norte do Rio Grande do Sul observou a importância dos hipertensos a serem orientados quanto à leitura adequada dos rótulos e adquirir alimentos com percentual menor que 5\% do valor diário para sódio em relação à recomendação máxima para um adulto, reforçando a importância da correta leitura dos rótulos. ${ }^{40}$

O termo de compromisso ${ }^{41}$ firmado entre o Ministério da Saúde e outras associações estabeleceu metas nacionais para redução do teor de sódio em alimentos processados no Brasil, dentre os quais estão inseridos o pão bisnaguinha e o pão francês. Observa-se que os pães integrais, indicados especialmente para doentes crônicos, não têm uma lei que limita o valor máximo de sódio para esse alimento.

Constatou-se, neste estudo, uma média de preço de $\mathrm{R} \$ 6,94$ (em relação a $500 \mathrm{~g}$ de pão). Considerando que uma pessoa compre, por mês, quatro pães - R \$27,76 mensais - isso irá representar um gasto de 3,83\% em relação ao salário mínimo (junho de 2014 - R \$ 724,00). Devese atentar, portanto, às gramagens dos pães, pois estes diferem em preço e quantidade. O que parece ser mais barato pode possuir menor gramagem, o que não é vantajoso financeiramente. Esta análise foi feita pelo dato de, na prática clínica, a questão do preço ser citada constantemente por pacientes como um impedimento para o consumo de pães integrais.

Em estudo de Pimentel \& Simões, ${ }^{35}$ encontrou-se baixo consumo de fibras, o que pode ser atribuído a seu elevado custo. Das entrevistas realizadas no estudo, $84 \%$ dos participantes concordaram que "os alimentos enriquecidos com fibras apresentam custo mais alto que os demais". Os resultados encontrados na pesquisa reforçam os dados encontrados no presente estudo, de que os alimentos integrais são onerosos aos olhos dos consumidores e que o consumo alimentar per capita por classes de rendimento está centrado em salários maiores de R \$ 1.089,00, segundo pesquisa do IBGE. $^{19}$

Através deste estudo, observou-se que a relação entre o preço e a adequação dos rótulos às leis vigentes reflete que quanto maior a quantidade de adequações às leis, maior é o valor do pão - ou seja, quanto mais adequações às leis, mais caro é o produto. Notou-se, também, que os rótulos que continham na informação nutricional a farinha integral como primeiro ingrediente são mais caros do que os que não o apresentavam $(\mathrm{p}<0,04)$. Além disso, constatou-se que os rótulos que possuíam o selo de certificação eram os mais caros em comparação com aqueles que não o possuíam.

Nesta pesquisa foram encontradas algumas dificuldades metodológicas, como a escassez de estudos semelhantes para comparação. As gramagens dos rótulos não são padronizadas em 500g, e foi necessário adequá-las em relação ao preço. Observou-se, criteriosamente, a quantidade das porções que também não são padronizadas em $50 \mathrm{~g}$, e as que não se enquadravam neste quesito foram excluídas da pesquisa. Da mesma forma, os rótulos que não possuíam a expressão "pão integral" fizeram com que algumas amostras fossem descartadas. 


\section{Conclusão}

Os rótulos de pães integrais avaliados neste estudo, em sua maioria, apresentaram diversas irregularidades em relação às legislações brasileiras vigentes e ao Substitutivo que está tramitando no Congresso Nacional.

A rotulagem nutricional é uma ferramenta fundamental que colabora para a escolha adequada dos alimentos. Diante dos resultados, fica evidente a necessidade de fiscalização dos órgãos competentes quanto ao cumprimento das normas estabelecidas, exigindo suas devidas adequações. Devido à importância dos pães integrais para a saúde do consumidor e sabendo que estes compõem a dieta de consumidores que possuem algumas patologias, é indispensável o rigor no cumprimento das leis.

O estudo colaborou para oferecer informações para que tanto o consumidor como os profissionais de saúde possam fazer melhores escolhas e indicações quanto a esse produto, assim como seus similares, pois o consumidor recebe informações não condizentes com a realidade dos rótulos.

Sugere-se a elaboração de mais estudos nesta área, com o objetivo de alertar e melhorar as orientações aos consumidores quanto aos pães integrais, para que eles possam utilizar o rótulo como uma ferramenta que colabore na escolha de alimentos benéficos à sua saúde.

Portanto, a partir dos resultados obtidos neste estudo, o profissional nutricionista pode recomendar a seus pacientes que as melhores escolhas de pães integrais são aquelas cujo rótulo apresenta o selo de certificação, observando as quantidades maiores de fibras e menores de sódio. A informação nutricional deve ser utilizada como uma ferramenta que auxilie nas escolhas, até que haja maior rigidez e cumprimento das leis vigentes e que sejam aplicadas penalidades a quem não se adequar a elas.

\section{Referências}

1. Paula TP, Steemburgo T, Almeida JC, Dall'Alba V, Gross JL, Azevedo MJ. The role of dietary approaches to stop hypertension (DASH): diet food groups in blood pressure in type 2 diabetes. Br. J. Nutr. 2012; 108(1):155-62.

2. Mello VD, Laaksonen DE. Fibras na dieta: tendências atuais e benefícios à saúde na síndrome metabólica e no diabetes tipo 2. Arq. Bras. Endocrinol. Metab. 2009; 53(5):509-518.

3. Steemburgo T, Dall'Alba V, Almeida JC, Zelmanovitz T, Gross JL, Azevedo MJ. Intake of soluble fibers has a protective role for the presence of metabolic syndrome in patients with type 2 diabetes. Eur. J. Clin. Nutr. 2009; 63(1):127-133. 
4. Anderson James W, Baird P, Davis RH Jr, Ferreri S, Knudtson M, Koraym A, Waters V. et al. Health benefits of dietary fiber. Nutrition Reviews 2009; 67(4):188-205.

5. Wu AH, Dwyer KM, Fan Z, Shircore A, Fan J, Dwyer JH. Dietary fiber and progression of atherosclerosis: the Los Angeles atherosclerosis Study. Am. J. Clin. Nutr. 2003; 78(6):1085-1091.

6. Mahan L, Kathllen, Escott-Stump S. K. Alimento, nutrição e dietoterapia. 11. ed. São Paulo: Roca; 2005.

7. El-Serag HB, Satia JA, Rabeneck L. Dietary intake and the risk of gastro-oesophageal reflux disease a cross sectional study in volunteers. Gastro. Hepatol. 2005; 54(1):11-17.

8. Wu AH, Tseng CC, Hankin J, Bernstein L. Fiber intake and risk of adrenocarcinoma of the esophagus and stomach. Cancer Causes Control 2007; 18(7):713-722.

9. Marlett JA, McBurney MI, Slavin JL. Position of the American Dietetic Association: health implications of dietary fiber. J. Am. Diet. Assoc. 2002; 102(7):993-1000.

10. Cade JE, Burley VJ, Greenwood DC. Dietary fibre and risk of breast cancer in the UK women's cohort study. Int. J. Epidemiol. 2007; 36(2):431-438.

11. Park Y, Brinton LA, Subar AF, Hollenbeck A, Schatzkin A. Dietary fiber intake and risk of breast cancer in postmenopausal women: the National Institutes of Health: AARP diet and health study. Am. J. Clin. Nutr. 2009; 90(3):664-671.

12. Rooney MC, Wald A. Interventions for the management of weight and body composition chances in women with breast cancer. Clin. J. Oncol. Nurs. 2007; 11(1):41-52.

13. Howarth NC, Saltzman E, Roberts SB. Dietary fiber and weight regulation. Nutrition Reviews 2001; 59(5):129-39.

14. Brasil. Portaria no 354, de 18 de julho de 1996. Aprova a Norma Técnica referente à Farinha de Trigo. Diário Oficial da União 22 jul. 1996.

15. American Association of Cereal Chemists International. Members agree on definition of whole grain [Internet]. [acesso 01 jun. 2010]. Disponível em: http://www.aaccnet.org/initiatives/definitions/ Documents/WholeGrains/wgflyer.pdf

16. United States Food and Drug Administration. FDA provides guidance on "Whole Grain" for manufacturers [Internet]. [acesso 01 jun. 2010]. Disponível em: http://www.fda.gov/NewsEvents/ Newsroom/PressAnnouncements/2006/ucm108598.htm

17. Whole Grains Council. What is a whole Grain? [Internet]. [acesso 05 maio 2014]. Disponível em: http://wholegrainscouncil.org/whole-grains-101/what-is-a-whole-grain

18. Brasil. Resolução RDC no 90, de 18 de outubro de 2000. Dispõe sobre o Regulamento Técnico para fixação de identidade e qualidade de pão. Diário Oficial da União 20 out. 2000.

19. Instituto Brasileiro de Geografia e Estatística. Pesquisa de orçamentos familiares 2008-2009: análise do consumo alimentar pessoal no Brasil. Rio de Janeiro: IBGE; 2011.

20. Brasil. Ministério da Saúde. Guia alimentar para a população brasileira: promovendo a alimentação saudável. Brasília: Ministério da Saúde; 2008. 
21. Brasil. Ministério da Saúde. Estratégias para o cuidado da pessoa com doença crônica. Brasília: Ministério da Saúde; 2014. 162 p. Cadernos de Atenção Básica, n.35.

22. Moratoya EE, Carvalhaes GC, Wander AE, Almeida LMMC. Mudanças no padrão de consumo alimentar no Brasil e no mundo. Rev. Política Agrícola 2013; 22(1):72-84.

23. Gaino NM, Amâncio RD, Oetterer M, Silva MV. Disponibilidade domiciliar de alimentos industrializados no Brasil. Hig. Alimentar 2012; 26(206/207):55-63.

24. Monteiro CA, Levy RB, Claro RM, Castro IRR, Cannon G. A new classification of foods based on the extent and purpose of their processing. Cad. Saúde Pública 2010; 26(11):2039-2049.

25. Popkin BM. Global nutrition dynamics: the world is shifting rapidly toward a diet linked with noncommunicable diseases. Am J. Clin. Nutr. 2006; 84(2):289-298.

26. Brasil. Ministério da Saúde. Guia alimentar para a população brasileira. 2 ed. Brasília: Ministério da Saúde; 2014.

27. Cotta RMM, Machado JC. Programa Bolsa Família e segurança alimentar e nutricional no Brasil: revisão crítica da literatura. Rev. Panam. Salud Publica. 2013:33(1):54-60.

28. Brasil. Resolução RDC n ${ }^{\circ}$ 359, de 23 de dezembro de 2003. Aprova Regulamento Técnico de Porções de Alimentos Embalados para Fins de Rotulagem Nutricional. Diário Oficial da União 26 dez. 2003.

29. Lobanco CM, Vedovato GM, Cano CB, Bastos DHM. Fidedignidade de rótulos de alimentos comercializados no município de São Paulo. Rev. Saúde Pública 2009; 43(3):499-505.

30. Brasil. RDC no 54, de 12 de novembro de 2012. Dispõe sobre o Regulamento Técnico sobre Informação Nutricional Complementar. Diário Oficial da União 19 nov. 2012.

31. Brasil. Projeto de Lei no 5.081-A, 2013. Dispõe sobre normas de comercialização de pão integral. Proposição sujeita à apreciação conclusiva pelas Comissões: Comissão de Desenvolvimento Econômico, Indústria e Comércio; Defesa do Consumidor;. Constituição e Justiça e de Cidadania Disponível em http: http://www.camara.gov.br/sileg/integras/1066871.pdf

32. Brasil. Congresso Nacional. Substitutivo ao Projeto de Lei no 5.081, de 2013. Dispõe sobre o uso da expressão "integral e afins" na rotulagem de alimentos à base de cereais. Projeto tramitando no Congresso Nacional para aprovação. Disponível em: http://www.camara.gov.br/proposicoesWeb/ fichadetramitacao?idProposicao $=600204$

33. Brasil. Resolução RDC no 259, de 20 de setembro de 2002. Aprova o Regulamento Técnico sobre Rotulagem de Alimentos Embalados. Diário Oficial da União 23 set. 2002.

34. PROTESTE Associação de Consumidores. Este pão é mesmo integral [Internet]. Disponível em http://www.proteste.org.br/alimentacao/nc/noticia/este-pao-e-mesmo-integral.

35. Pimentel TC, Simões GS. Percepção dos consumidores em relação às fibras alimentares e seus produtos. Revista Brasileira de Pesquisa em Alimentos 2012; 3(1):11-18.

36. Whole Grains Council. About Us? [Internet]. [acesso em 05 maio 2014. Disponível em: http:// wholegrainscouncil.org/about-us.

37. Whole Grains Council. Stamp FAQ Manufacturers [Internet]. [acesso em 05 maio 2014] .Disponível em http://wholegrainscouncil.org/whole-grain-stamp/stamp-faq-manufacturers 
38. Whole Grains Council. Whole Grain Stamp? [Internet]. [acesso em 05 maio 2014]. Disponível em http://wholegrainscouncil.org/whole-grain-stamp

39. Whole Grains Council. It's Working [Internet]. [acesso em 05 maio 2014. Disponível em: http:// wholegrainscouncil.org/whole-grain-stamp/its-working

40. Orlando R, Pinheiro TLF, Volkweis DSH, Colussi El. Avaliação da alimentação e sua relação com as doenças crônicas não transmissíveis de um grupo de idosos de um município da região norte do estado do Rio Grande do Sul. Revista de Enfermagem 2010-2011; 6-7(6-7)203-217. [acesso em 23 maio 2014]. Disponível em: http://revistas.fw.uri.br/index.php/revistadeenfermagem/article/view/736

41. Brasil. Termo de Compromisso que firmam entre si a União, por intermédio do Ministério da Saúde e a Associação Brasileira das Indústrias de Alimentação - ABIA, com a finalidade de estabelecer metas nacionais para a redução do teor de sódio em alimentos processados no Brasil. Brasília, 28 ago. 2012. Disponível em: http://189.28.128.100/dab/docs/portaldab/documentos/termo_6_ago_2012.pdf 
